\title{
The Clinical Features of Graves' Orbitopathy with Elevated Intraocular Pressure
}

\author{
Shu-Xian Fan $\left(\mathbb{D},{ }^{1}\right.$ Peng Zeng $\left(\mathbb{D},{ }^{1}\right.$ Zi-Jing Li $\mathbb{D},{ }^{1}$ Jing Wang $\left(\mathbb{D},{ }^{1}\right.$ Jia-Qi Liang $\left(\mathbb{D},{ }^{1}\right.$ \\ Yun-Ru Liao $\mathbb{D}^{1},{ }^{1}$ Yu-Xin $H u\left(\mathbb{D},{ }^{1}\right.$ Ming-Tong Xu $\mathbb{D}^{2},{ }^{2}$ and Mei Wang $\mathbb{D}^{1}$ \\ ${ }^{1}$ Department of Ophthalmology, Sun Yat-sen Memorial Hospital, Sun Yat-sen University, Guangzhou 510120, China \\ ${ }^{2}$ Department of Endocrinology, Sun Yat-sen Memorial Hospital, Sun Yat-sen University, Guangzhou 510120, China \\ Correspondence should be addressed to Mei Wang; wangmei2@mail.sysu.edu.cn
}

Received 1 July 2020; Revised 31 October 2020; Accepted 13 January 2021; Published 25 January 2021

Academic Editor: Takayuki Baba

Copyright (C) 2021 Shu-Xian Fan et al. This is an open access article distributed under the Creative Commons Attribution License, which permits unrestricted use, distribution, and reproduction in any medium, provided the original work is properly cited.

Background. To investigate the clinical characteristics of Graves' orbitopathy (GO) with elevated intraocular pressure (IOP) using the European Group of Graves' Orbitopathy (EUGOGO) system. Methods. In this retrospective study, the clinical data of GO patients with elevated IOP $(\geq 21 \mathrm{mmHg})$ were collected in Sun Yat-sen Memorial Hospital from January 2010 to June 2016. The demographic characteristics, clinical history of thyroid disease and GO, and ocular examination data were evaluated, and the activity and severity of GO were classified. Results. Data were collected from 58 eyes of 39 patients. The durations of thyroid disease and GO were $15.9 \pm 18.9$ months and $7.5 \pm 6.2$ months, respectively. The average IOP was $24.8 \pm 5.3 \mathrm{mmHg}$ (range: $21-55 \mathrm{mmHg}$ ). No significant difference in IOP was observed between active and inactive eyes. Eight eyes (13.8\%), 29 eyes (50.0\%), and 21 eyes (36.2\%) were graded as mild, moderate-severe, and sightthreatening disease, respectively, according to the EUGOGO classification. The IOP was not significantly different among the three EUGOGO grades. No glaucomatous optic nerve damage or visual field defects were found. Conclusion. Increased IOP was evident for every grade of GO severity and activity of the EUGOGO system. IOP, glaucomatous optic nerve damage, and visual fields must be evaluated regularly during follow-up evaluations, regardless of the degree of activity and severity of GO.

\section{Background}

Graves' orbitopathy (GO), also known as thyroid-related ophthalmopathy, is an autoimmune disease characterized by enlarged extraocular muscles and increased retrobulbar fat. The pathogenesis of GO is associated with lymphocyte infiltration and glycosaminoglycan deposits in the extraocular muscles, connective tissue, and orbital fat [1], which may cause clinical ophthalmopathy, including proptosis, diplopia, periorbital edema, corneal breakdown, and compressive optic neuropathy [2]. Elevated intraocular pressure (IOP) in GO was first reported by Wessely in 1918 [3], and since then, many studies have been conducted on the incidence and treatment of increased IOP [4-7]. Although elevated IOP directly associated with GO is a less common problem, it may result in damage to the optic nerve [8].
No physical signs or symptoms, only signs, soft-tissue involvement, proptosis, extraocular muscle signs, corneal involvement, and sight loss (NOSPECS) and the European Group of Graves' Orbitopathy (EUGOGO) classifications are popular grading systems for assessing the clinical severity of GO. However, the clinical characteristics of the elevated IOP have mainly been analyzed using the NOSPECS classification of GO, and few reports describing increased IOP in GO using the EUGOGO system are currently available. In this study, we analyzed elevated IOP in GO using the EUGOGO system and determined its relationship to data in the NOSPECS system.

1.1. Patients and Methods. The study was conducted in Sun Yat-sen Memorial Hospital, Sun Yat-sen University, Guangzhou, China, from January 2010 to June 2016. This 
study was conducted in accordance with the tenets of the Declaration of Helsinki, and the protocol of this study was approved by the Sun Yat-sen University Sun Yat-sen Memorial Hospital Ethical Committee in China. GO was diagnosed in the Department of Endocrinology and the Department of Ophthalmology based on clinical and laboratory findings [9]. The clinical data of 39 GO patients with elevated IOP $(\geq 21 \mathrm{mmHg})$ and complete examinations were collected. Patients using any IOP-lowering agents or ocular drops that influence the ocular surface and those with a history of prior ophthalmic surgery, orbital decompression, or other immune disease were excluded.

The collected data included age, sex, signs, symptoms, duration of GO, clinical activity, and severity classification of GO according to the EUGOGO system, history and duration of thyroid disease, history of smoking, and presence of other systemic and eye diseases. Ocular examinations included best-corrected visual acuity, slit-lamp examination of the anterior segment, and fundus examination with $a+90$ lens. Proptosis was measured with the Hertel exophthalmometer. IOP measurements were performed in the primary position using a noncontact tonometer (NCT) (Canon TX-20, YZB/ JAP3501-2012, Tokyo, Japan) or the Schiotz tonometer in cases of strabismus; each eye was measured 3 times and took the average in the morning time. Standard automated visual fields were obtained using the Humphrey automated visual field analyzer (program 30-2).

Glaucomatous changes in the optic nerve were defined as a cup disc ratio greater than 0.6 , vertical cup asymmetry greater than 0.2 , neuroretinal rim loss or notching with or without disc hemorrhages, and nerve fiber layer defects. According to the probability plot, visual fields were considered abnormal if 2 or more adjacent points were depressed $\geq 5 \mathrm{~dB}$ or if 1 point was depressed $\geq 10 \mathrm{~dB}$ compared to the surrounding points in any region of the central 30 degrees of the visual field, with a mean deviation greater than $2 \mathrm{~dB}$, pattern standard deviation outside the $95 \%$ confidence interval, and a glaucoma hemifield test result outside the normal range.

Two grading systems were used to assess the severity of GO: the NOSPECS classification, which was described by Werner [10], and the EUGOGO classification [11], which includes the categories of mild, moderate-severe, and sightthreatening disease. The mild disease was defined as minor lid retraction $(<2 \mathrm{~mm})$, mild soft-tissue involvement, exophthalmos $<3 \mathrm{~mm}$ above normal value for race and gender, no or intermittent diplopia, and corneal exposure that is responsive to lubricants. Moderate-severe GO was defined as lid retraction $\geq 2 \mathrm{~mm}$, moderate or severe soft-tissue involvement or exophthalmos $\geq 3 \mathrm{~mm}$ above normal value for race and gender, and variable or constant diplopia. Sightthreatening GO was defined as dysthyroid optic neuropathy and/or severe corneal exposure (large epithelial and/or stromal defects) or corneal breakdown (descemetocele or frank perforation).

The activity of GO is measured by the clinical activity score (CAS) [11], which includes spontaneous retrobulbar pain, pain on attempted upward or downward gaze, redness of the eyelids, redness of the conjunctiva, swelling of the caruncle or plica, swelling of the eyelids, and swelling of the conjunctiva (chemosis). A CAS $\geq 3 / 7$ is indicative of positive activity.

1.2. Statistical Analysis. Statistical analyses were performed using SPSS (Statistical Package for Social Sciences; SPSS Inc. IBM, Armonk, NY) version 22.0. Data are expressed as the means \pm standard deviation. The significance of differences in the mean IOP according to gender, eye, and disease activity, and the mean proptosis value of the eye was assessed by $t$-tests (independent samples $t$-test or $t^{\prime}$ test). The Kruskal-Wallis test was used to evaluate significant differences in the mean IOP according to the EUGOGO classification and the proptosis classification in the NOSPECS system. Differences were considered statistically significant at $P \leq 0.05$.

\section{Results}

2.1. Demographic and Other Characteristics. A total of 58 eyes of 39 GO patients were included in the analyses. The demographic and clinical patient characteristics, including the affected eye, gender, age, course of thyroid disease and GO, and smoking habits, are summarized in Table 1. Male patients (21 patients, $53.8 \%$ ) were more than female patients (18 patents, $46.2 \%)$. The mean age was $49.8 \pm 12.5$ years, and male ( $49.5 \pm 12.5$ years) and female $(50.2 \pm 10.4$ years $)$ patients' mean age had no significant difference $(P=0.109)$. The most common primary thyroid disease was hyperthyroidism (36 patients, 92.3\%). The average durations of thyroid disease and GO were $15.9 \pm 18.9$ months and $7.5 \pm 6.2$ months, respectively. All patients with smoking habits were male. Most patients (28 patients, $71.8 \%$ ) used antihyperthyroidism medication, and others had undergone treatments, including radioiodine therapy (7 patients) and thyroidectomy (4 patients). However, many patients (34 patients, $87.2 \%$ ) still had hyperthyroidism, with only $17.9 \%$ of the patients exhibiting normal thyroid function at the time of ocular examination. No patients had received systemic corticosteroid treatment or orbital radiotherapy before the ocular examination.

2.2. Ocular Presentation. The mean IOP was $24.8 \pm 5.3 \mathrm{mmHg}$ (range: $21-55 \mathrm{mmHg}$ ). No significant difference in IOP was observed between genders or eyes $(P=0.109, P=0.739$; Figure 1$)$. The mean IOP of smokers $(27.38 \pm 8.76 \mathrm{mmHg})$ was higher than that of nonsmokers $(23.73 \pm 2.45 \mathrm{mmHg}, \quad P<0.00)$. Twenty-four eyes $(41.1 \%)$ were active, with a CAS $\geq 3$, and the remaining eyes were inactive, with a CAS $<3$. The mean IOP in the active eyes was $25.98 \pm 7.56 \mathrm{mmHg}$, which was not significantly different from that in the inactive eyes $(24.02 \pm 2.79 \mathrm{mmHg}$, $P=0.237$ ) (Figure 1).

One patient was found to have disc swelling, but no glaucomatous optic nerve damage or visual field defects, such as arcuate scotoma, were found in any patients. All cup disc ratios in eyes with increased IOP were less than 0.6. 
TABle 1: Demographic characteristics of elevated intraocular pressure of GO patients.

\begin{tabular}{lc}
\hline Characteristic & \\
\hline Gender (male/female) & $21 / 18$ \\
Eye (right/left) & $28 / 30$ \\
Smoking (yes/no) & $10 / 29$ \\
Age (mean \pm SD, range, years) & $49.8 \pm 12.5(25-75)$ \\
Male patients & $49.5 \pm 14.4(25-75)$ \\
Female patients & $50.2 \pm 10.4(27-67)$ \\
Duration (mean \pm SD, range, months) & \\
Thyroid disease & $15.9 \pm 18.9(1-96)$ \\
Graves' orbitopathy & $7.5 \pm 6.2(1-36)$ \\
Diagnosis of primary thyroid disease (number, \%) & $36(92.3)$ \\
Hyperthyroidism & $1(2.6)$ \\
Thyroid cancer & $2(5.1)$ \\
Euthyroidism. & $28(71.8)$ \\
Treatment of thyroid disease (number, \%) & $7(17.9)$ \\
Antihyperthyroidism drugs & $4(10.3)$ \\
I ${ }^{131}$ & \\
Thyroidectomy & $1(2.6)$ \\
Current thyroid function (number, \%) & $34(87.2)$ \\
Normal & $3(7.7)$ \\
Hyperthyroidism & $1(2.6)$ \\
Hypothyroidism &
\end{tabular}

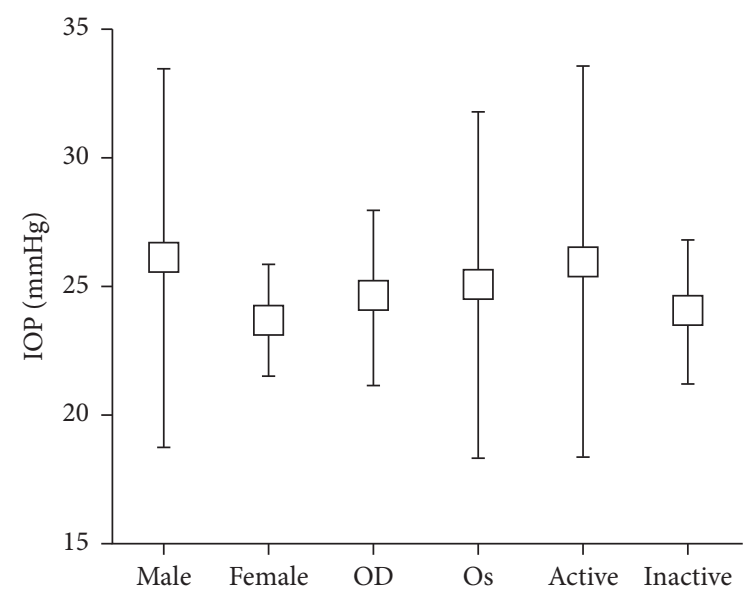

FIgURe 1: Box and whisker plot showing the distribution of intraocular pressure (IOP) for different genders, eyes, and disease activity grades. No significant difference in IOP was found between genders, eyes, and disease activity grades.

The distribution of eyes with elevated IOP was different according to the EUGOGO and NOSPECS systems. In the EUGOGO classification, eyes with elevated IOP were distributed among all scopes of severities, including grades of mild ( 8 eyes, $13.8 \%$ ), moderate-severe ( 29 eyes, $50.0 \%$ ), and sight-threatening (21 eyes, 36.2\%). However, in the NOSPECS system, the distribution was mainly concentrated in phase 4 (36 eyes, 27 patients, 64.1\%) and phase 6 (20 eyes, 13 patients, $34.5 \%)$. Most of the eyes in the mild or moderate-severe GO categories according to the EUGOGO system were classified as phase 4 and phase 6 according to the NOSPECS system (Figure 2). In addition, the IOP levels

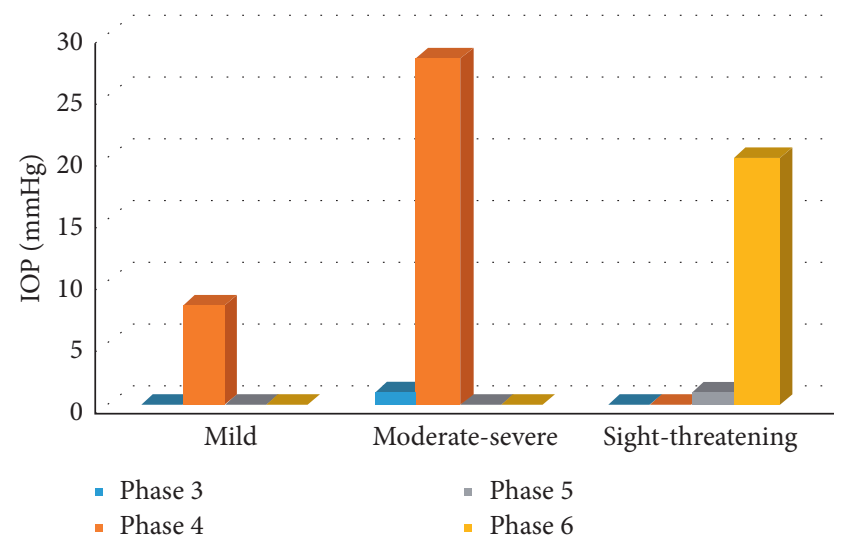

Figure 2: $3 \mathrm{D}$ column chart comparing the distribution of high IOP in the NOSPECS and EUGOGO systems.

were not significantly different among various severities of the EUGOGO or NOSPECS system (Table 2).

The mean proptosis value in all eyes with increased IOP was $19.8 \pm 3.2 \mathrm{~mm}$ (range: $14-28 \mathrm{~mm}$ ), and the mean values were $19.9 \pm 3.1 \mathrm{~mm}$ (range: $15-28 \mathrm{~mm}$ ) in right eyes and $19.9 \pm 3.3 \mathrm{~mm}$ (range: $14-28 \mathrm{~mm}$ ) in left eyes. The exophthalmos degree was not significantly different between the right and left eyes. According to the classification of proptosis in the NOSPECS system [12], the IOP gradually increased with increased proptosis, although no statistically significant association was found between these factors (Table 3).

\section{Discussion}

This study aimed to explore the clinical features of elevated IOP in GO according to the EUGOGO system. The study revealed that elevated IOP was present in eyes with each severity degree of the EUGOGO system, from mild to sightthreatening, but it was more common in the higher stages of the NOSPECS system, including phases 4 and 6 . Elevated IOP was present in both active and inactive eyes, and the mean IOP in the inactive eyes was comparable to that in the active eyes.

Similar to other reports $[13,14]$, the average age of all patients was $49.8 \pm 12.5$ years, and the average IOP was $24.8 \pm 5.3 \mathrm{mmHg}$ in this study. Cockerham et al. $[14,15]$ reported that the IOP in GO was greater than $22 \mathrm{mmHg}$ but less than $30 \mathrm{mmHg}$. However, the present study identified 6 patients with IOP levels greater than $30 \mathrm{mmHg}$, and the highest IOP recorded was $55 \mathrm{mmHg}$, as measured with an NCT. This discrepancy may be due to differences in sample sizes, races, and the pathophysiology of IOP increases. Although elevated IOP is common in GO patients [8], the optic disc or visual field damage characteristics of glaucoma were not observed in this study.

Similar to the study of Sara et al., there were more male patients than female patients [16]. In our study, the range of IOP was wider for male patients and left eyes maybe because there were more male patients and more left eye cases. 
TABLE 2: Comparison of intraocular pressure (IOP) in severities of Graves' orbitopathy.

\begin{tabular}{lcc}
\hline & Number of eyes & IOP $(\mathrm{mmHg})$ \\
\hline Mild & 8 & $23.72 \pm 3.33$ \\
Moderate-severe & 29 & $25.57 \pm 7.04$ \\
Sight-threatening & 21 & $24.24 \pm 2.55$ \\
$\mathrm{P}^{*}$ & $/$ & 0.417 \\
Phase 3 & 1 & 22 \\
Phase 4 & 36 & $25.25 \pm 6.49$ \\
Phase 5 & 1 & 22 \\
Phase 6 & 20 & $24.35 \pm 2.56$ \\
P & $/$ & 0.463 \\
\hline
\end{tabular}

* The Kruskal-Wallis test; independent samples $t^{\prime}$ test, data of IOP in phases 3 and 5 deleted for statistics due to too little sample size in them.

TABle 3: Comparison of elevated intraocular pressure (IOP) in different degrees of proptosis.

\begin{tabular}{lcc}
\hline Degree of exophthalmos & Number of eyes $(\%)$ & IOP $(\mathrm{mmHg})$ \\
\hline Absent $($ normal $-20 \mathrm{~mm})$ & $32(55.2)$ & $23.88 \pm 2.65$ \\
Mild $(21 \mathrm{~mm}-23 \mathrm{~mm})$ & $15(25.9)$ & $24.93 \pm 5.05$ \\
Moderate $(24 \mathrm{~mm}-27 \mathrm{~mm})$ & $5(8.6)$ & $25.60 \pm 4.72$ \\
Severe $(\geq 28 \mathrm{~mm})$ & $6(10.3)$ & $29.00 \pm 12.85$ \\
$\mathrm{P}^{*}$ & $/$ & 0.721 \\
\hline
\end{tabular}

* The Kruskal-Wallis test.

This study showed that many eyes classified as phase 4 (36 eyes, 64.1\%) and phase 6 (20 eyes, 34.5\%) in the NOSPECS system exhibited elevated IOP, which is similar to other reports of proptosis and ocular movement disorders in GO accompanied by elevated IOP [8, 13]. However, the distribution of high IOP was wider, ranging from mild to sight-threatening, in the EUGOGO system. In addition, most eyes with high IOP in the mild and moderate-severe categories were classified as phase 4 in the NOSPECS system, which suggests that the use of both the EUGOGO and NOSPECS systems is beneficial for monitoring IOP in clinical practice. The IOP should also be monitored, even in mild cases of GO, according to the EUGOGO classification, especially when extraocular muscles are involved according to the NOSPECS classification.

In this study, elevated IOP was rarely observed in NOSPECS phase 3 eyes (only 1 eye), which is slightly different from the report by Behrouzi [8], who found that IOP increases, including open-angle glaucoma and suspected glaucoma, were not uncommon in phase 3 eyes, in addition to being present in phase 4 and phase 6 eyes.

In this study, increased IOP was observed not only in the active eyes but also in the inactive eyes. Behrouzi et al. [8] and Konuk et al. [17] also found that IOP may remain elevated despite disease remission. Additionally, chronic and persistent episcleral venous pressure may be elevated due to secondary changes in these structures [18] or due to a compromised aqueous drainage system [8]. The finding that IOP in the inactive eyes was similar to that in the active eyes in this study profoundly supports the above presumption.

Cockerham et al. $[8,13]$ showed that ophthalmometric values were $>20 \mathrm{~mm}$ in $60-77 \%$ of GO patients. Other reports have shown that the extent of IOP elevation may be correlated with the degree of proptosis $[19,20]$. This study also showed that IOP elevation tended to be associated with increased proptosis.

No glaucomatous optic changes or visual field defects were detected in this study. Forte et al. [21] did not find glaucomatous perimetric defects either; however, they found glaucomatous excavation of the optic disc and retinal nerve fiber layer thinning in some cases of GO with intraocular hypertension using OCT. In addition, Behrouzi et al. [8] reported visual field changes in some cases of compressive optic neuropathy with high IOP. All of the above data indicate that persistent IOP elevation may result in the progression of glaucomatous neuropathy. The absence of glaucomatous optic changes and visual field defects in this study may be due to the insufficient duration of elevated IOP for the manifestation of optic nerve damage.

Goldmann applanation tonometry (GAT) is the gold standard for measuring IOP. However, an NCT was used in this study. The NCT utilizes an air jet to achieve corneal flattening based on the same principle that GAT applies to measure IOP [22]. The main advantages of an NCT over GAT include that it is noninvasive and more convenient, thus facilitating patient cooperation, and no direct contact with the cornea occurs, which decreases its consequences $[23,24]$ and lessens any influence on tear film instability in GO patients [25]. In addition, many studies have reported no significant differences between IOP measurements obtained by GAT and an NCT $[24,26]$. In another study, a Tono-Pen tonometer was also used to measure IOP in GO patients [27].

\section{Conclusions}

In conclusion, increased IOP was present in eyes with every degree of severity and disease activity of GO in the EUGOGO system, which showed a broader distribution than the NOSPECS system. Although no glaucomatous optic changes or visual field defects were detected in this study, routine IOP measurements and ophthalmic examinations are recommended to identify glaucomatous damage during follow-up examinations, regardless of the activity and severity of GO.

\section{Abbreviations}

GO: Graves' orbitopathy

IOP: Intraocular pressure

EUGOGO: European Group of Graves' Orbitopathy

CAS: $\quad$ Clinical activity score

NCT: Noncontact tonometer

GAT: Goldmann applanation tonometry.

\section{Data Availability}

The datasets used and/or analyzed during the current study are available from the corresponding author upon reasonable request. 


\section{Ethical Approval}

This study was conducted in accordance with the tenets of the Declaration of Helsinki, and the protocol of this study was approved by the Sun Yat-sen University's Sun Yat-sen Memorial Hospital Ethical Committee in China.

\section{Disclosure}

The authors confirm that the work described has not been published before, and the publication has been approved by the responsible authorities at the institution where the work is carried out.

\section{Conflicts of Interest}

The authors declare that they have no conflicts of interest.

\section{Authors' Contributions}

SF and PZ contributed equally to this work. SF, PZ and WM drafted the manuscript and participated in information collection and editing of the manuscript. ZL, JW, JL, YL, YH, MX were resposible for data collection. All authors read and approved the final manuscript

\section{References}

[1] T. Shanmuganathan, C. Girgis, H. Lahooti, B. Champion, and J. R. Wall, "Does autoimmunity against thyroglobulin play a role in the pathogenesis of Graves' ophthalmopathy: a review," Clinical Ophthalmology, vol. 9, pp. 2271-2276, 2015.

[2] O. C. Guler, C. Onal, and G. Arslan, "Graves' ophthalmopathy treated with protracted hypofractionated low-dose adaptive radiotherapy: case report and review of the literature," Wiener Klinische Wochenschrift, vol. 128, no. 7-8, pp. 299-303, 2016.

[3] K. Wessely, "Discussion of Hertel E. WeitererBetragzurLehre von Augendruck," Der Dtsch Ophthlmol Ges, vol. 41, pp. 80-81, 1918.

[4] A. J. Otto, L. Koornneef, M. P. Mourits, and L. Deen-van Leeuwen, "Retrobulbar pressures measured during surgical decompression of the orbit," British Journal of Ophthalmology, vol. 80, no. 12, pp. 1042-1045, 1996.

[5] D. R. Fishman and S. C. Benes, "Upgaze intraocular pressure changes and strabismus in Graves' ophthalmopathy," Journal of Clinical Neuro-Ophthalmology, vol. 11, no. 3, pp. 162-165, 1991.

[6] R. A. Saunders, E. M. Helveston, and F. D. Ellis, "Differential intraocular pressure in strabismus diagnosis," Ophthalmology, vol. 88 , no. 1 , pp. 59-70, 1981.

[7] I. Goldberg, "Thyroid eye disease and glaucoma," Journal of Glaucoma, vol. 12, no. 6, pp. 494-496, 2003.

[8] Z. Behrouzi, H. M. Rabei, F. Azizi et al., "Prevalence of openangle glaucoma, glaucoma suspect, and ocular hypertension in thyroid-related immune orbitopathy," Journal of Glaucoma, vol. 16, no. 4, pp. 358-362, 2007.

[9] G. B. Bartley and C. A. Gorman, "Diagnostic criteria for Graves' ophthalmopathy," American Journal of Ophthalmology, vol. 119, no. 6, pp. 792-795, 1995.

[10] S. C. Werner, "Modification of the classification of the eye changes of Graves' disease," American Journal of Ophthalmology, vol. 83, no. 5, pp. 725-727, 1977.

[11] L. Bartalena, L. Baldeschi, K. Boboridis et al., "The 2016 European thyroid association/European Group on Graves' orbitopathy guidelines for the management of Graves' orbitopathy," European Thyroid Journal, vol. 5, no. 1, pp. 9-26, 2016.

[12] D. H. J. L. Van, “Orbital Graves' disease,” Ophthalmology, vol. 88 , no. 6 , pp. 479-483, 1981.

[13] R. Kalmann and M. P. Mourits, "Prevalence and management of elevated intraocular pressure in patients with Graves' orbitopathy," British Journal of Ophthalmology, vol. 82, no. 7, pp. 754-757, 1998.

[14] K. P. Cockerham, C. Pal, B. Jani, A. Wolter, and J. S. Kennerdell, "The prevalence and implications of ocular hypertension and glaucoma in thyroid-associated orbitopathy," Ophthalmology, vol. 104, no. 6, pp. 914-917, 1997.

[15] J. H. Norris, J. J. Ross, M. Kazim, D. Selva, and R. Malhotra, "The effect of orbital decompression surgery on refraction and intraocular pressure in patients with thyroid orbitopathy," Eye, vol. 26, no. 4, pp. 535-543, 2012.

[16] S. Gharib, Z. Moazezi, and M. Ali Bayani, "Prevalence and severity of ocular involvement in Graves' disease according to sex and age: a clinical study from Babol, Iran," Caspian Journal of Internal Medicine Spring, vol. 9, no. 2, pp. 178-183, 2018.

[17] O. Konuk, Z. Onaran, S. Ozhan Oktar, C. Yucel, and M. Unal, "Intraocular pressure and superior ophthalmic vein blood flow velocity in Graves' orbitopathy: relation with the clinical features," Graefe's Archive for Clinical and Experimental Ophthalmology, vol. 247, no. 11, pp. 1555-1559, 2009.

[18] I. Haefliger, G. V. Arx, and A. R. Pimentel, "Pathophysiology of intraocular pressure increase and glaucoma prevalence in thyroid eye disease: a mini-review," Klinische Monatsblätter für Augenheilkunde, vol. 227, no. 3, pp. 292-293, 2010.

[19] R. S. Manor, O. Kurz, and Z. Lewitus, "Intraocular pressure in endocrinological patients with exophthalmos," Ophthalmologica, vol. 168, no. 4, pp. 241-252, 1974.

[20] P. Pohjanpelto, "The thyroid gland and intraocular pressure. Tonographic study of 187 patients with thyroid disease," Acta Ophthalmologica (Copenh), vol. 97, no. 1, pp. 1-70, 1968.

[21] R. Forte, P. Bonavolontà, and P. Vassallo, "Evaluation of retinal nerve fiber layer with optic nerve tracking optical coherence tomography in thyroid-associated orbitopathy," Ophthalmologica, vol. 224, no. 2, pp. 116-121, 2010.

[22] M. B. Shields, "The non-contact tonometer. Its value and limitations," Survey of Ophthalmology, vol. 24, no. 4, pp. 211-219, 1980.

[23] C. Kirwan, M. O’Keefe, and B. Lanigan, “Corneal hysteresis and intraocular pressure measurement in children using the reichert ocular response analyzer," American Journal of Ophthalmology, vol. 142, no. 6, pp. 990-992, 2006.

[24] K. C. Ogbuehi, "Assessment of the accuracy and reliability of the Topcon CT80 non-contact tonometer," Clinical and Experimental Optometry, vol. 89, no. 5, pp. 310-314, 2006.

[25] P. Novaes, A. B. Diniz Grisolia, and T. J. Smith, "Update on thyroid-associated Ophthalmopathy with a special emphasis on the ocular surface," Clinical Diabetes and Endocrinology, vol. 2, p. 19, 2016.

[26] A. Zareei, M. Razeghinejad, M. Nowroozzadeh, Y. Mehrabi, and M. Aghazadeh-Amiri, "Intraocular pressure measurement by three different tonometers in primary congenital glaucoma," Journal of Ophthalmic and Vision Research, vol. 10, no. 1, pp. 43-48, 2015.

[27] I. Rahman, P. S. Cannon, and S. A. Sadiq, "Tonopen versus Goldmann applanation tonometry for detecting restrictive thyroid eye disease," Ophthalmic Plastic \& Reconstructive Surgery, vol. 26, no. 1, pp. 36-38, 2010. 